\title{
TADs assisted Comouflage Orthodontic Treatment of Class II Division 1 Malocclusion in a Non Growing Patient: A case report
}

Dr Ankita Gupta,' Dr Trilok Shrivastava²

1Consultant Orthodontist, Department of Orthodontics, Cure Dent Smile designing center, Pune, India, 2Reader, Department of Orthodontics, People's Dental Academy, Bhopal, India

Correspondence: Dr. Ankita Gupta; Email: doctor.ankitagupta@gmail.com

\section{ABSTRACT}

Class II, Division I malocclusion has been described as the most frequent treatment problem in orthodontic practice. Aim \& objectives of the present case report was to evaluate the management of skeletal Class II division 1 malocclusion in non growing patient with extraction of upper first premolars. Clinical and cephalometric evaluation revealed skeletal Class II with Angles Class II division 1 malocclusion with mild mandibular anterior crowding and increased overjet, severe maxillary incisor proclination, mild mandibular crowding, exaggerated curve of spee, convex profile, incompetent lips, increased overjet and overbite. Maxillary first premolars were extracted followed by en-masse retraction of anteriors with the help of temporary anchorage devices (TADS) to avoid anchorage loss. Mandibular incisor was extracted to correct curve of spee. Following treatment marked improvement in patient's smile, facial profile and lip competence were achieved and there was a remarkable increase in the patient's confidence and quality of life.

Keywords: Anchorage loss, Class II divl malocclusion, Non growing patient, TADs (temporary anchorage devices).

\section{INTRODUCTION}

Non-growing patients with skeletal Class II malocclusions can be treated by only two possible treatment approaches: (1) orthodontic camouflage, based on selective extraction of permanent teeth followed by retraction of the protruding maxillary incisors to improve both dental occlusion and facial aesthetics without correcting the underlying skeletal problem; or (2) orthognathic surgery to reposition the mandible or the maxilla. Skeletal Class II problems are due to mandibular deficiency or downwardbackward rotation of the mandible caused by excessive vertical growth of the maxilla. Surgical treatment, therefore, consists of mandibular advancement, superior repositioning of the maxilla, or a combination. Mandibular deficiency is the problem in about two thirds of surgical patients; one third require maxillary surgery, either alone (15\%) or combined with mandibular surgery $(20 \%)$. $^{1}$

Premolars are probably the most commonly extracted teeth for orthodontic purposes as they are located between the anterior and posterior segments. For correction of Class II malocclusions in non-growing patients extractions can involve 2 maxillary premolars ${ }^{2}$ or 2 maxillary and 2 mandibular premolars ${ }^{3}$ The extraction of only 2 maxillary premolars is generally indicated when there is no crowding or cephalometric discrepancy in the mandibular arch. ${ }^{4,5}$ Studies have shown that patient satisfaction with camouflage treatment is similar to that achieved with surgical mandibular advancement ${ }^{6}$ and that treatment with two maxillary premolar extractions gives a better occlusal result than treatment with four premolars extractions.

Anchorage control plays a pivotal role in the effective management of orthodontic patients for obtaining both structural and facial esthetics. Anchorage is defined as the resistance to unwanted tooth movement7,8 or as the desired reaction of posterior teeth to space closure mechanotherapy. Obtaining maximum or absolute anchorage has always been an ultimate goal for the practicing orthodontist, often resulting in a condition, called anchorage loss. Anchorage loss is the reciprocal reaction of the anchor unit that can obstruct the success of orthodontic treatment by complicating anteroposterior correction. 'To address this problem, many appliances and techniques have been devised; Nance holding arch, transpalatal bars, extraoral traction, multiple ${ }^{10}$ teeth at the anchorage segment, and differential moments ${ }^{11,12}$ are some commonly used ones. However, all these methods have a few inherent disadvantages-complicated designs, need for exceptional patient cooperation, elaborate wire bending, and so on. 
In past few years, titanium screws have gained enormous popularity in the orthodontic community and are being considered as absolute sources of orthodontic anchorage. ${ }^{13-15}$ Their primary advantages are easy placement and removal, immediate loading, placement at various anatomic locations including alveolar bone between roots of teeth, and low cost. These screws have spawned many clinical applications, such as en-masse retraction of anterior teeth.

Lower incisor extraction can be regarded as a valuable option in the pursuit of excellence in orthodontic results in terms of function, aesthetics and stability. This treatment option is also indicated in Class II Division 1 skeletal and dental malocclusions with maxillary protrusion and crowding or protrusion of the lower incisors. Typically, lower incisor extraction should be associated with the extraction of maxillary premolars while keeping the Class II molar relationship but establishing normal canine occlusion.

\section{CASE REPORT}

A 19-year-old male patient came for orthodontic treatment with convex facial profile, incompetent lips, acute nasolabial angle and symmetrical face. Retrognathic mandible was observable. Intraoral examination revealed severely proclined maxillary anteriors, increased overjet with mandibular mild anterior crowding with increased curve of spee. Molars and canines were in Class II relation. (Figure 1). Cephalometric analysis showed skeletal Class II with retrognathic mandible with low angle (Figure 2).

The primary treatment objectives were to reduce the increased overjet, levelling of exaggerated curve of spee, and improve the smile of patient. Patient was banded and bonded with 0.022 slot MBT prescribed bracket in both arches. Maxillary first premolars in both the quadrants were extracted to gain spaces required for retracting anteriors. Lower incisor was extracted to reduce mild crowding and level curve of spee in lower arch. Leveling and alignment was started with the use of $0.014 \mathrm{NiTi}, 0.016 \mathrm{NiTi}$ with lacebacks and bendbacks to prevent anchorage loss. In mandibular arch, incisors were intruded, to correct deep bite by intrusion arch (0.017 x 0.025 TMA) and activated by a molar tip back bend.
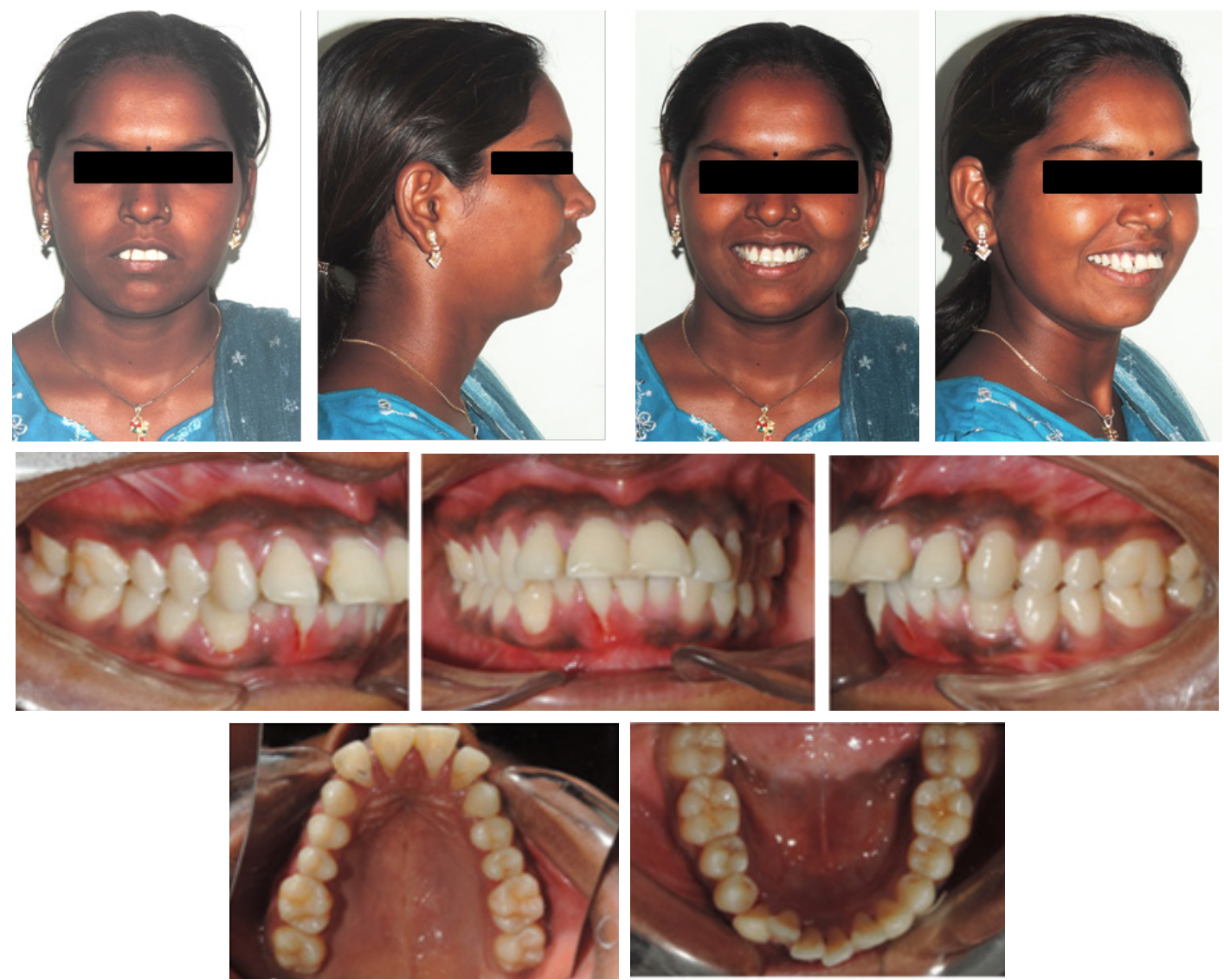

Figure 1: Pre-treatment photographs 


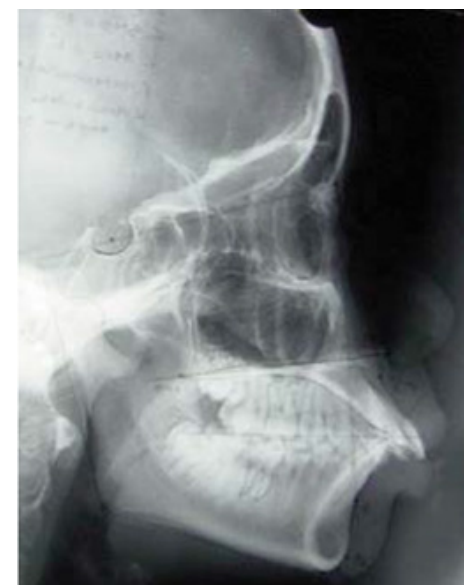

Figure 2: Pre-treatment cephalograph

After the initial levelling and aligning, TADs $(1.5 / 8 \mathrm{~mm})$ were placed between second premolar and first molar for anterior retraction in first and second quadrants and extraction spaces were closed using power chains. Final settling of occlusion was done with proper interdigitation, inclination, angulation, ideal overjet and overbite. Debonded and retention was given by fixed retainers and upper \& lower Hawley's retainers. Patient was advised to follow up in retention period. (Figure 3-6)
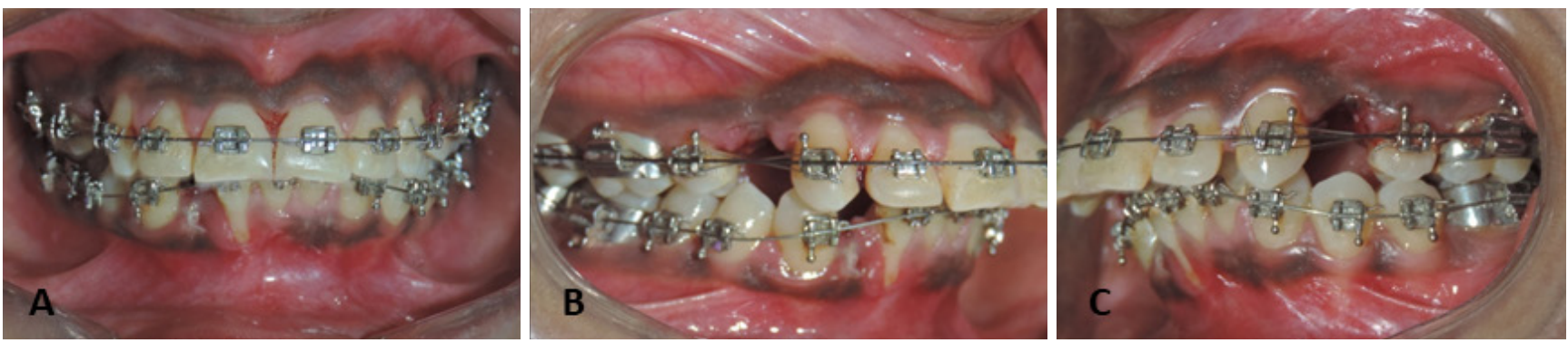

Figure 3: A, B and C - Leveling and Alignment-022 Edgewise Bracket system placed with 0.014 NiTi archwire
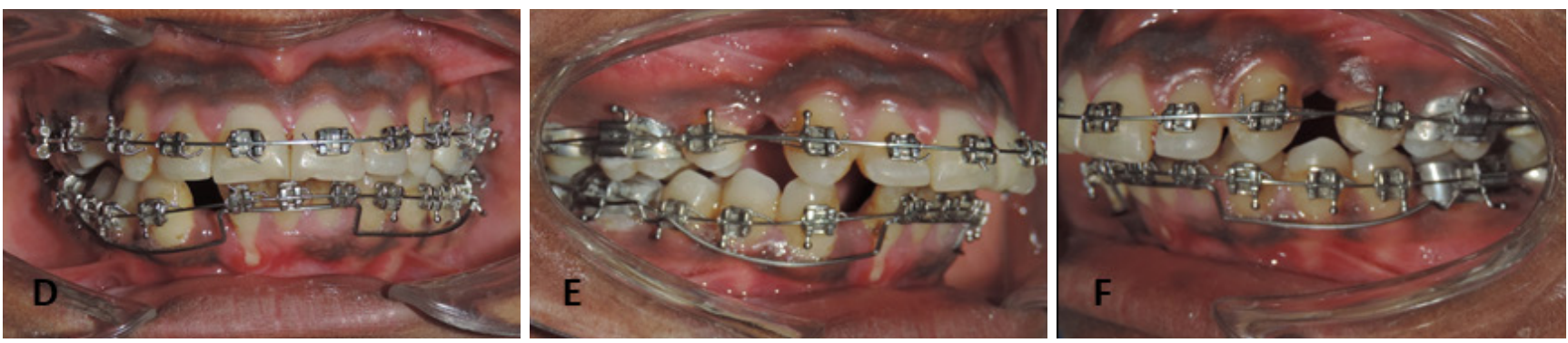

Figure 4: D,E and F - Leveling and Alignment Follow up 0.016 NiTi and Intrusion arch 0.017 × 0.025 TMA wire
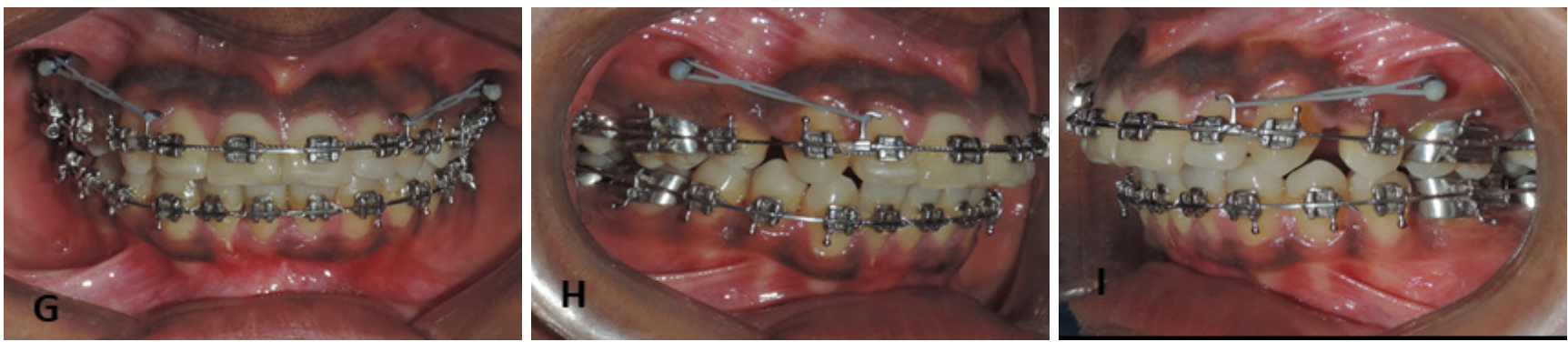

Figure 5: G,H and I - Anterior retraction with TADs $0.019 \times 0.025$ SS 

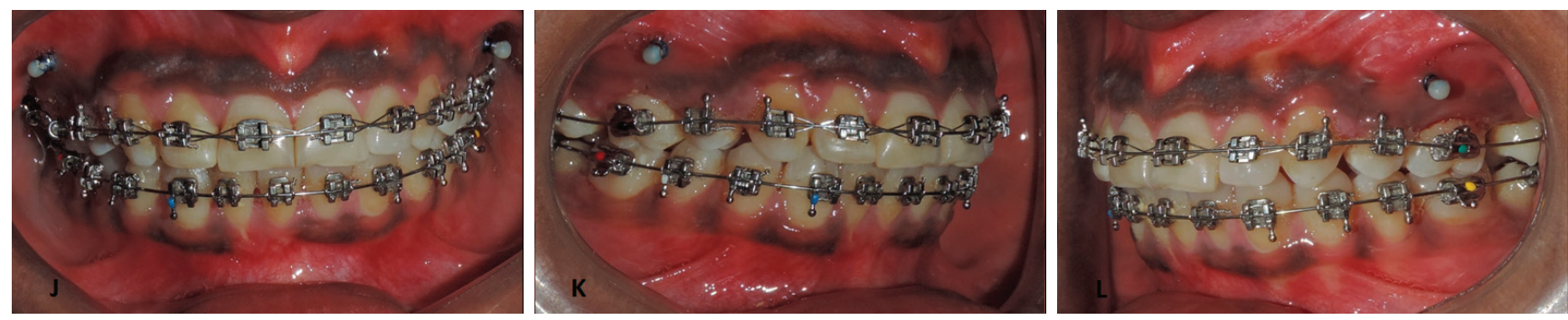

Figure 6: J, K and L - After 6 months of space closure

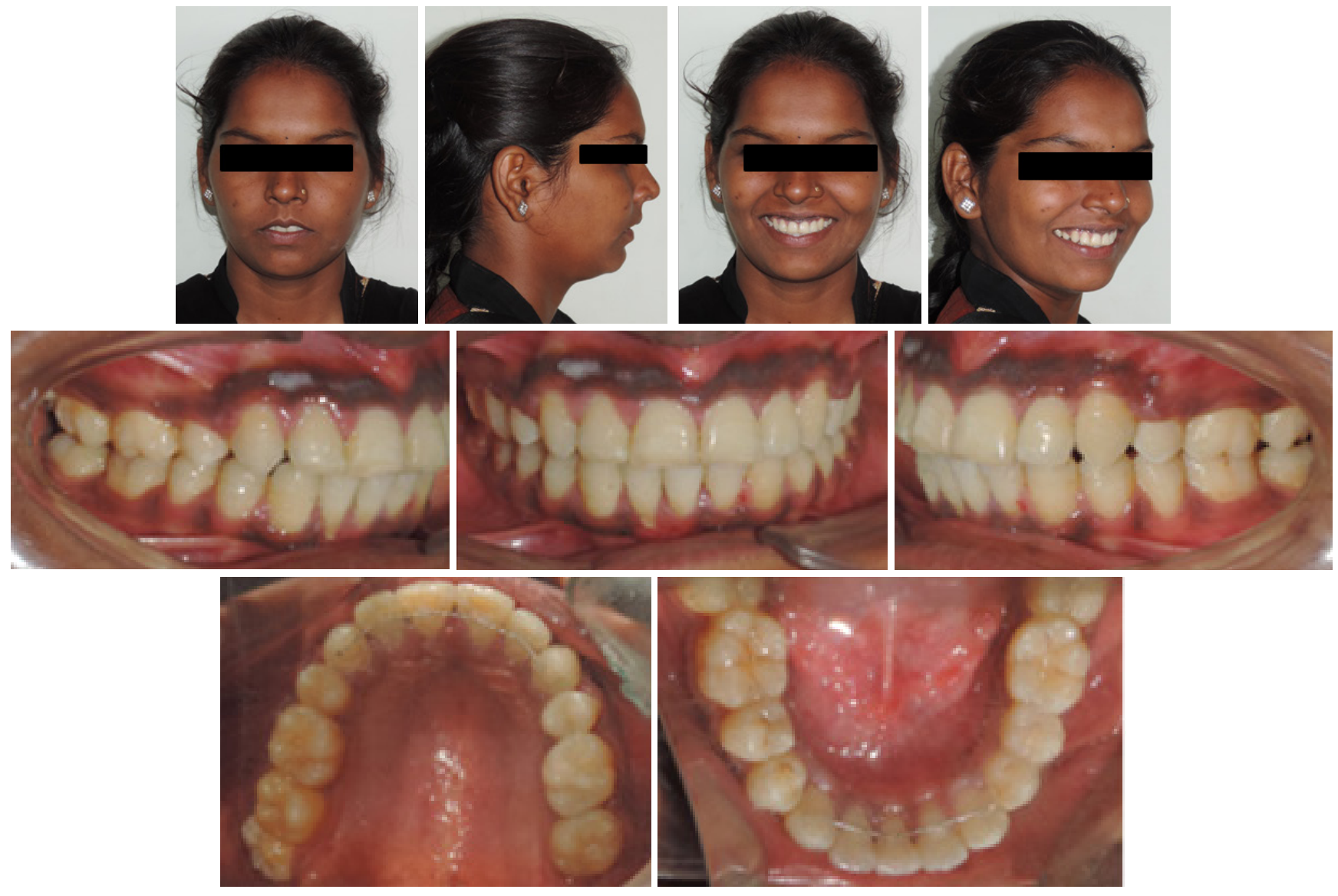

Figure 7: Post-treatment photographs

\section{Post treatment assessment}

Lip competence and a straight profile were achieved, improving the patient's facial appearance. A functional occlusion with normal overjet and overbite; (Figure 7). Duration of the treatment was 21 months. The patient and her parents were very happy with complete satisfaction.

\section{DISCUSSION}

Patient had improved smile and profile after orthodontic treatment. Upper incisors were retracted to achieve normal incisor inclinations, overjet and overbite. Bilateral Class I canine relation was achieved with maximum intercuspation. The case was successfully managed by contemporary orthodontic technique with TADs without the anchorage loss.

\section{CONCLUSION}

Camouflage treatment of Class II malocclusion in adults is challenging. Extractions of premolars, if undertaken after a thorough diagnosis often leads to positive profile changes and an overall satisfactory facial esthetics. A well chosen individualized treatment plan, undertaken with sound biomechanical principles and appropriate control of orthodontic mechanics to execute the plan is the surest way to achieve predictable results with minimal side effects. 


\section{REFERENCES}

1. Proffit WR, White RP, Sarver DM. Contemporary treatment of dentofacial deformity. St. Louis: Mosby; 2003.

2. Cleall JF, Begole EA. Diagnosis and treatment of Class II Division 2 malocclusion. Angle Orthod 52:38-60; 1982.

3. Strang RHW. Tratado de ortodoncia. Buenos Aires: Editorial Bibliogra'fica Argentina; 1957. p. 560-70, 657-71.

4. Bishara SE, Cummins DM, Jakobsen JR, Zaher AR. Dentofacial and soft tissue changes in Class II, Division 1 cases treated with and without extractions. Am J Orthod Dentofacial Orthop 107:28-37; 1995.

5. Rock WP. Treatment of Class II malocclusions with removable appliances. Part 4. Class II Division 2 treatment. Br Dent J 168:298-302;1990.

6. Mihalik, C.A.; Proffit, W.R.; and Phillips, C.: Long-term followup of Class Il adults treated with orthodontic camouflage: A comparison with orthognathic surgery outcomes, Am. J. Orthod. 123:266-278, 2003.

7. Lindquist JT. The edgewise appliance. In: Graber TM, editor. Orthodontics: current principles and techniques. St Lovis:Mosby; 1985. p. 565-640.

8. Proffit WR. Biomechanics and mechanics. In: Proffit WR, Fields HW Jr, editors. Contemporary orthodontics. St Louis: Mosby; 2000. p. 295362.

9. Geron S, Shpack N, Kandos S, Davidovitch M, Vardimon AD.Anchorage loss-a multifactorial response. Angle Orthod 2003; 73:730-7.

10. Renfroe EW. The factor of stabilization in anchorage. Am J Orthod 1956; 42:86-97.

11. Hart A, Taft L, Greenberg SN. The effectiveness of differential moments in establishing and maintaining anchorage. Am J Orthod Dentofacial Orthop 1992; 102:434-42.

12. Rajcich MM, Sadowsky C. Efficacy of intra-arch mechanics using differential moments for achieving anchorage control in extraction cases. Am J Orthod Dentofacial Orthop 1997; 112:441-8.

13. Costa A, Raffaini M, Melsen B. Miniscrews as orthodontic anchorage: a preliminary report. Int J Adult Orthod Orthognath Surg 1998; 13:201-29.

14. Park HS, Bae SM, Kyung HM. Micro-implant anchorage for treatment of skeletal Class I bialveolar protrusion. J Clin Orthod 2001; 35:417-22.

15. Lee JS, Park HS, Kyung HM. Micro-implant for lingual treatment of a skeletal Class II malocclusion. J Clin Orthod 2001;35: 643-7 